\title{
Towards Topological Quasi-Freestanding Stanene via Substrate Engineering
}

\author{
Domenico Di Sante, ${ }^{1}$ Philipp Eck, ${ }^{1}$ Maximilian Bauernfeind,${ }^{2}$ Marius Will, ${ }^{2}$ \\ Ronny Thomale, ${ }^{1}$ Jörg Schäfer, ${ }^{2}$ Ralph Claessen, ${ }^{2}$ and Giorgio Sangiovanni ${ }^{1}$ \\ ${ }^{1}$ Institut für Theoretische Physik und Astrophysik, Universität Würzburg, \\ Am Hubland Campus Süd, Würzburg 97074, Germany* \\ ${ }^{2}$ Physikalisches Institut and Röntgen Research Center for Complex Material Systems, \\ Universität Würzburg, Am Hubland Campus Süd, Würzburg 97074, Germany
}

(Dated: November 7, 2018)

\begin{abstract}
In search for a new generation of spintronics hardware, material candidates for room temperature quantum spin Hall effect (QSHE) have become a contemporary focus of investigation. Inspired by the original proposal for QSHE in graphene, several heterostructures have been synthesized, aiming at a hexagonal monolayer of heavier group IV elements promoting the QSHE bulk gap via increased spin-orbit coupling. So far, the monolayer/substrate coupling, which can manifest itself in strain, deformation, and hybridization, has proven to be detrimental to the aspired QSHE conditions for the monolayer. For stanene, the Sn analogue of graphene, we investigate how an interposing buffer layer mediates between monolayer and substrate in order to optimize the QSHE setting. From a detailed density functional theory study, we highlight the principal mechanisms induced by such a buffer layer to accomplish quasi-freestanding stanene in its QSHE phase. We complement our theoretical predictions by presenting the first attempts to grow a buffer layer on $\mathrm{SiC}(0001)$ on which stanene can be deposited.
\end{abstract}

\section{INTRODUCTION}

Quantum spin Hall (QSH) systems are twodimensional bulk non-conducting materials featuring topologically non-trivial conducting edge modes, whose stability against external perturbations is ensured by time reversal symmetry [1, 2]. After the first theoretical prediction in graphene by Kane and Mele [3, 4] and the subsequent prediction and experimental realization in $\mathrm{HgTe} / \mathrm{CdTe}$ quantum wells [5, 6, it became evident that the small bulk energy gap represents the hardest obstacle to render QSH materials operative at room temperature and, as such, relevant to technological applications.

An initial plausible way of increasing the bulk gap is to enhance the spin-orbit coupling strength of the constituting atoms. Within the group-IV elements, freestanding honeycomb-like structures made by $\mathrm{Si}, \mathrm{Ge}$, and $\mathrm{Sn}$ atoms, dubbed silicene, germanene, and stanene, respectively, were estimated in theory to exhibit gaps up to $100 \mathrm{meV}$ 7 9 . A realistic implementation, however, always requires the stabilization of freestanding 2D layers on a supporting substrate, with no guarantee that the resulting symmetry breaking keeps the QSH phase intact. For instance, this detrimental effect to the QSHE phase is seen when an insulating $\mathrm{MoS}_{2}$ substrate stabilizes the growth of germanene [10 or a $\mathrm{Bi}_{2} \mathrm{Te}_{3}$ template is used to accommodate stanene flakes [11. In both cases, the topological bulk gap does not survive the interaction with the substrate, and either yields a metallic or trivially insulating monolayer.

By contrast, a constructive effect of the substrate is

*Electronic address: domenico.disante@physik.uni-wuerzburg.de observed for bismuthene on $\mathrm{SiC}$ 12. There, the strong hybridization between the substrate and the $\mathrm{Bi} \pi$ orbitals leaves a sizable topological bulk gap of $0.8 \mathrm{eV}$, highlighting bismuthene as the first material realization of a QSH system operable at ambient temperature. Monolayer $\mathrm{WTe}_{2}$ is another recent example where the interaction between the $2 \mathrm{D}$ layer and the substrate stabilizes the QSH phase by opening a finite gap, thus avoiding contributions from the bulk material which may be detrimental to the edge conductivity 13 15.

On the basis of these observations, it is evident that any meaningful theoretical investigation must properly take the role of substrates into consideration. With a particular focus on stanene, several studies have attempted to include these effects [11, 16 19, while only a few of them have considered technologically achievable, and hence relevant, substrates [18, 19]. Rather, our objective is to address commercial wafers of wide-gap semiconductors as possible substrate candidates, in order to follow the motif of reaching proposals that might allow for the integration of QSH physics into ambient life devices. $\mathrm{SiC}(0001)$ turns out to be a promising way along this direction 20. However, dangling bond passivation is a well-known problem of semiconductor surfaces. The presence of highly reactive surface charge may be detrimental to the QSH phase. Recent theoretical investigations proceeded by removing the effect of the dangling bonds via hydrogen $(\mathrm{H})$ saturation [18, 21. Despite its modeling efficiency, this cannot be considered a viable solution, since no evidence of a $2 \mathrm{D}$ material grown on a pure $\mathrm{H}$-saturated surface has so far been reported in the literature.

For silicene and germanene, several studies report on attempts of reducing the interaction with the substrate 22 27. Regarding carbon, one monolayer deposited on $\mathrm{SiC}$ does not form graphene, as the symmetries and elec- 
tronic properties are destroyed by the strong binding. Moving towards almost freestanding graphene, one can either grow further carbon on this first layer, which in fact takes care of saturating the dangling bonds, or intercalate "spacers" such as $\mathrm{H}, \mathrm{Au}$ or O between the first layer and the substrate 28 34. Here, we explore another possibility, namely realizing the QSH phase in stanene on $\mathrm{SiC}(0001)$ by the insertion of a buffer layer of group-III and group- $\mathrm{V}$ elements. This way, the valence electrons from the buffer atoms saturate the $\mathrm{SiC}$ dangling bonds, keeping at the same time a favorable lattice matching. The positive effect of the buffer is counterbalanced by an unavoidable monolayer/substrate hybridization which, as we will explicate in the following, turns out not to damage the QSHE under particular conditions, and instead to even structurally stabilize stanene.

For buckled geometries, as it is also the case for stanene, a concomitant staggered potential (Semenoff mass [35]) emerges, which for specific buffers is strong enough to prevent the QSH formation [3]. Via a proper choice of the buffer characteristics, we tune the quantitative impact of the Semenoff mass term. While our DFT calculations show that on $\mathrm{Al}, \mathrm{Ga}$, In, and Tl, stanene is topologically trivial, we successfully minimize the detrimental effect of the Semenoff mass through switching to buffers made of group- $\mathrm{V}$ elements. Our calculations indeed demonstrate that the topology in stanene can be comfortably stabilized for buffer layers made of $\mathrm{P}$ and As atoms, which might stimulate further experimental progress along this direction.

\section{METHODS}

For our theoretical study of stanene on buffered $\mathrm{SiC}(0001)$ we employ state-of-the-art first-principles calculations based on density functional theory as implemented in the Vienna ab-initio simulation package (VASP) 36, within the projector-augmented-plane-wave (PAW) method [37, 38]. The generalized gradient approximation as parametrized by the PBE-GGA functional for the exchange-correlation potential is used [39], as well as different flavours of revised and van der Waals corrected functionals 40 43, by expanding the KohnSham wavefunctions into plane-waves up to an energy cut-off of $300 \mathrm{eV}$. We sample the Brillouin zone on an $8 \times 8 \times 1$ regular mesh, and if applicable, spin-orbit coupling (SOC) is self-consistently included [4]. Even though the stanene low-energy models are extracted by projecting onto Sn $p_{z^{-}}$and $s p_{2}$-type maximally localized Wannier functions (MLWF) by using the WANNIER90 package [45], we compute the $\mathbb{Z}_{2}$ topological invariant following the general method by Soluyanov and Vanderbilt [46] as in the Z2Pack code. That is, without relying on any pre-computed low-energy model, we directly compute the Wilson loop of the entirely occupied ab-initio spectrum [47. Phonon dispersions are obtained within the context of the frozen phonon method 48] as imple- a)
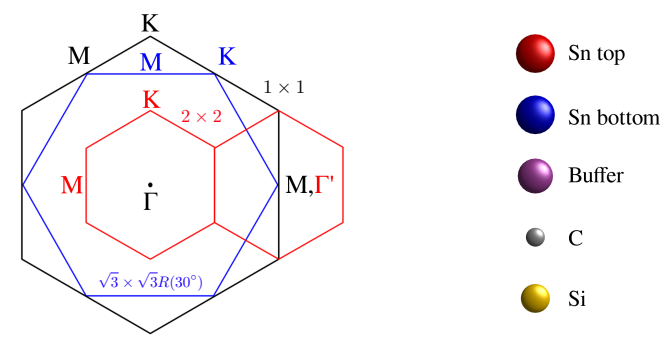

b)
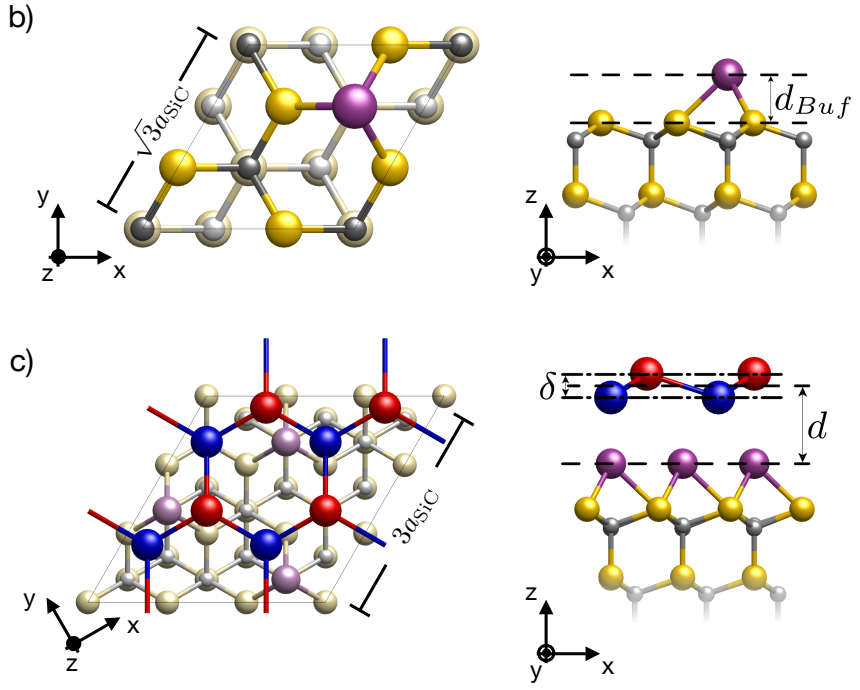

FIG. 1: a) Brillouin zones of $\sqrt{3} \times \sqrt{3} R\left(30^{\circ}\right) \mathrm{SiC}$ (blue), $1 \times 1$ stanene (black) and $2 \times 2$ stanene (red), with relative high symmetry points. b) Top and side views of the crystalline structure of buffered $\sqrt{3} \times \sqrt{3} R\left(30^{\circ}\right) \mathrm{SiC}$ and c) $2 \times 2$ stanene $/ 3 \times 3$ buffered $\mathrm{SiC}$. The bottom termination of the slab models is artificially passivated by $\mathrm{H}$ atoms. In the top view in panel c) the colors of the substrate are intentionally less intense compared to panel b) in order to emphasize the hexagonal lattice of $2 \times 2$ stanene.

mented in the PHONOPY code [49] with a $2 \times 2 \times 1$ supercell (as explained in the next paragraph, the unit cell consists already of $3 \times 3 \mathrm{SiC}$ and $2 \times 2$ stanene superstructures).

Each atom from group III and V can saturate three dangling bonds from the $\mathrm{SiC}$ surface, either from $\mathrm{Si}$ atoms of the $\mathrm{Si}$-terminated $\mathrm{SiC}$ or from $\mathrm{C}$ atoms from the C-terminated $\mathrm{SiC}$, as shown in Fig. 1b). This requires a minimal $\sqrt{3} \times \sqrt{3} R\left(30^{\circ}\right)$ reconstruction, whose Brillouin zone and crystal structure are reported in Fig. 1 1 ). Moreover, a $2 \times 2$ hexagonal lattice of stanene $(a=9.28$ $\AA$ ) turns out to have a good commensuration with a $3 \times 3$ reconstruction of $\mathrm{SiC}$ (lateral compressive strain $\varepsilon \sim 0.62 \%$ ), suggesting a promising strain-free overlayer deposition. A $3 \times 3$ buffered $\mathrm{SiC}$ template ultimately shows at its surface a triangular lattice of buffer atoms (see Fig. 1. $)$.

The large structural reconstructions enforce a folding of the electronic states into the supercell Brillouin zones, which map onto the primitive $1 \times 1$ Brillouin zones as sketched in Fig 1a. It is usually simpler to achieve 


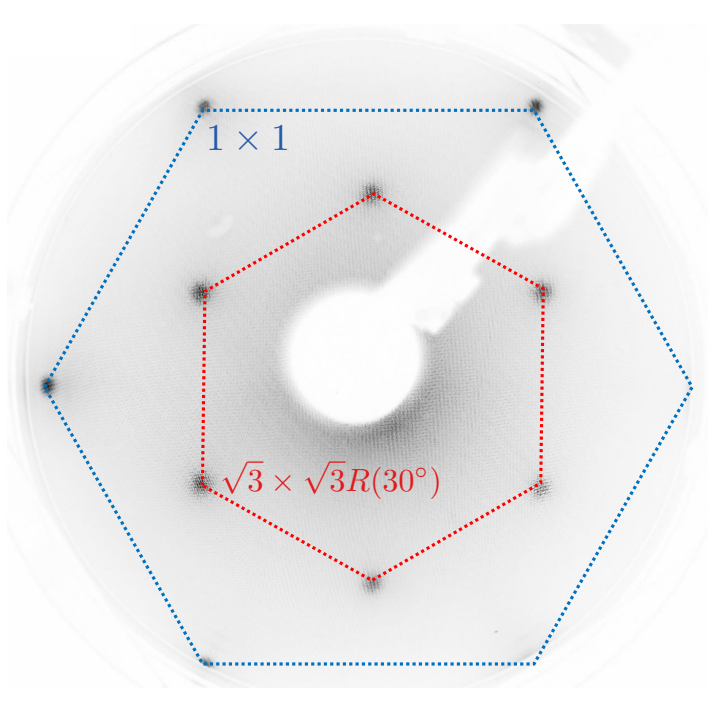

FIG. 2: LEED image of $\mathrm{Al} \sqrt{3} \times \sqrt{3} R\left(30^{\circ}\right)$ on Si-terminated $4 \mathrm{H}-\mathrm{SiC}(0001)$ recorded with an electron energy of $40 \mathrm{eV}$. Hexagons in red and blue highlight the Al induced $\sqrt{3} \times$ $\sqrt{3} R\left(30^{\circ}\right)$ and $1 \times 1$ spots, respectively.

a transparent physical description in the latter setting, where the unfolded band structure readily compares with the freestanding models when the symmetry breaking induced by the reconstruction is weak. The unfolding procedure we adopt in this work follows the lines described in Refs. 50, 51.

For the experimental realization of a buffer layer we use $n$-doped (0.01-0.03 $\Omega \mathrm{cm})$ Si-terminated $4 \mathrm{H}-\mathrm{SiC}(0001)$ wafer pieces. To prepare an atomically smooth, wellordered substrate surface on large-scales, the wafer pieces undergo a dry-etching process in a helium diluted hydrogen atmosphere with a flow of 2 standard litre per minute at 950 mbar and temperatures around $1200{ }^{\circ} \mathrm{C}$ for roughly 10 minutes. Subsequently, the H-terminated $\mathrm{SiC}(0001)$ samples 21] were transferred to the preparation chamber (base pressure $\mathrm{p}<3 \times 10^{-11}$ mbar) using a vacuum suitcase. Surface quality was inspected in situ by low-energy electron diffraction (LEED).

Prior to the epitaxial growth of $\mathrm{Al}$, the $\mathrm{H}$ termination of $\mathrm{SiC}(0001)$ has to be removed. This is achieved by heating the substrate to $\sim 620^{\circ} \mathrm{C}$ with a subsequent cooling to $\sim 350^{\circ} \mathrm{C}$ in the $\mathrm{Al}$ beam of the effusion cell, to form the $\mathrm{Al} \sqrt{3} \times \sqrt{3} R\left(30^{\circ}\right)$ lattice, as shown in Fig. 2. where LEED clearly shows the reconstruction of the commensurate buffer layer. All samples were heated by direct current and the temperatures were measured pyrometrically.

\section{STANENE ON GROUP-III BUFFER}

\section{A. Buffer analysis}

We consider $\mathrm{Al}, \mathrm{Ga}$, In, and $\mathrm{Tl}$ as group III elements of the periodic table, which all share the same $s^{2} p^{1}$ valence electronic configuration. It has been shown that $\mathrm{Al}$ and other group III atoms can saturate the (111) surface of a silicon crystal, inducing a $\sqrt{3} \times \sqrt{3} R\left(30^{\circ}\right)$ reconstruction 52 55. The underlying mechanism is that the three $\mathrm{Al}$ valence orbitals saturate three of the $\mathrm{Si}$ surface dangling bonds, which naturally leads to a $\sqrt{3} \times \sqrt{3}$ coverage of the Si substrate. By analogy, and based on the similarities that the $\mathrm{SiC}$ electronic structure shares with $\mathrm{Si}$, we assume here that the deposition of group-III atoms induces on the $\mathrm{SiC}$ surface the same $\sqrt{3} \times \sqrt{3} R\left(30^{\circ}\right)$ reconstruction as on silicon. Our theoretical assumption is indeed experimentally proven here for the first time for the case of an $\mathrm{Al}$ buffer on SiC. Fig. 2 shows a clear $\sqrt{3} \times \sqrt{3} R\left(30^{\circ}\right)$ reconstruction (red hexagon) on an underlying $1 \times 1$ template (blue hexagon).

The $s p^{3}$ orbitals of $\mathrm{Al}$ on $\mathrm{SiC}$ host the three valence electrons. The fourth one is empty and of $s p_{z}$-type character and gives rise to an antibonding in-gap state. The resulting band structures for this type of buffer layers on $\mathrm{SiC}$ (Si-face) are shown in Fig. 3a). The energy position of the antibonding in-gap state anticorrelates with the bonding distance of the buffer atom to $\mathrm{SiC}$, as we show in Fig. 3p). The lighter the buffer atom the shorter is the resulting distance, with the concomitant upward level repulsion of the antibonding orbital. This anticorrelation trend is independent of the $\mathrm{SiC}$ termination, holding both for the Si- and for the C-terminated surface.

In Fig. 3 a, in the case of the $\mathrm{Al}$ buffer on the Si face, in addition to the equilibrium $\mathrm{Al}-\mathrm{SiC}$ distance $d_{\mathrm{Buf}}=1.7 \AA$, we show the bonding-antibonding in-gap states with predominant buffer character for two more values of $d_{\text {Buf. }}$. If we lift $\mathrm{Al}$ to distances close to those obtained for $\mathrm{Tl}$, the bonding-antibonding states are located at a very similar position with a comparable gap $\Delta$ (green curve). On the other hand, by pushing the buffer artificially close to $\mathrm{SiC}\left(d_{\mathrm{Buf}}=1.3 \AA\right.$, red curve $), \Delta$ increases, with the antibonding state merging into the $\mathrm{SiC}$ conduction band. This evidences a rather weak dependence of the buffer states on the atomic species, proving that they instead decisively depend on $d_{\text {Buf }}$.

The energy position of the antibonding buffer level, and in turn the excitation gap, affect the strength of the hybridization when we add the stanene monolayer on top: The larger the gap of the $\mathrm{SiC}+$ buffer system is, the more strongly the buffer (conduction and valence) states hybridize with $\mathrm{SiC}$. This implies that for a large $\Delta$, the corresponding buffer states penetrate more pronouncedly into the bulk, and their surface localization is reduced. Stanene will hence be less affected in this situation. In the opposite case of a narrow gap, with buffer states situated at low energies, these have more weight at the surface and we expect sizable interaction effects between the buffer and stanene. A further important consequence is that top and bottom Sn atoms of the buckled inversionbreaking geometry have significantly distinct energy levels, as they are differently exposed to a strong interface potential. In Sec. $[\mathrm{IV}$ we are going to show explicitly that this directly translates into the presence of a staggered 

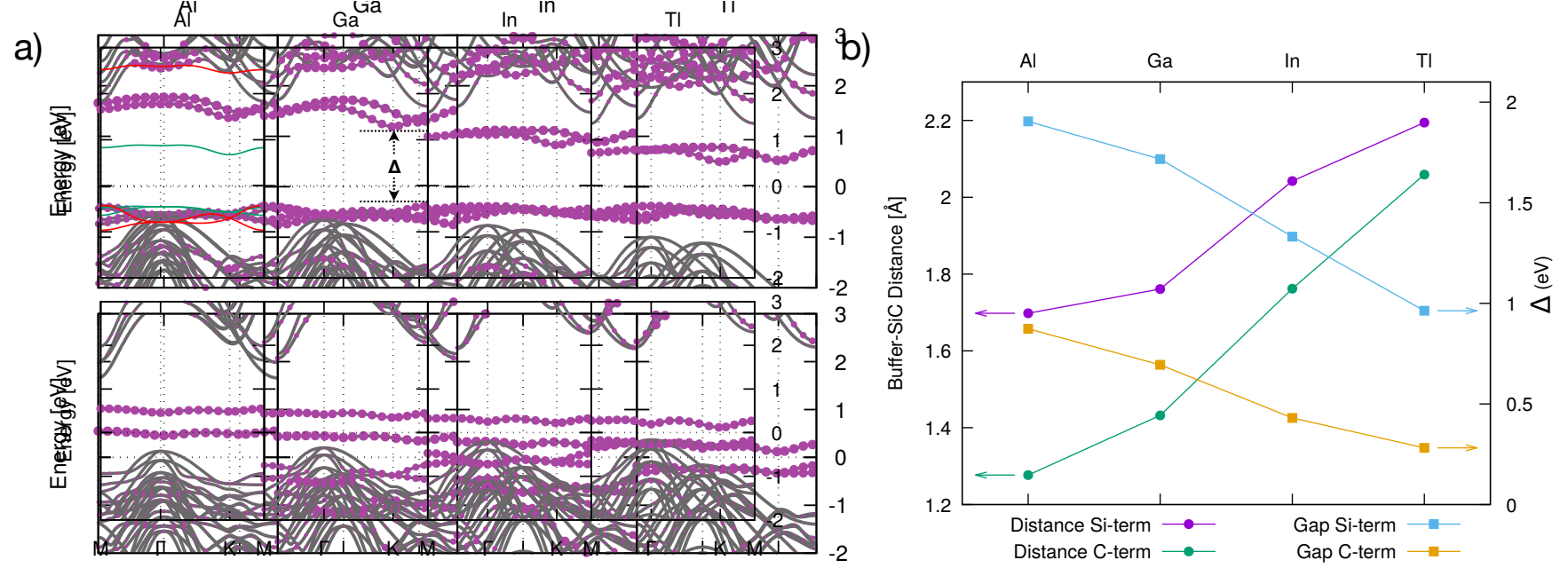

FIG. 3: a) Electronic band structures for group III buffered SiC (top row for Si-face and bottom row for C-face SiC) along the high-symmetry lines of the $\sqrt{3} \times \sqrt{3} R\left(30^{\circ}\right)$ Brillouin zone (see Fig. 1 1 ). The coloured dots highlight the orbital contribution from the buffer atom. For Al we additionally show the dependence of the bonding-antibonding in-gap states on the Al-SiC distance $d_{\mathrm{Buf}}$ : green corresponds to $d_{\mathrm{Buf}}=2.1 \AA$, i.e. roughly the same equilibrium distance obtained for Tl, while red corresponds to an exaggeratedly small value $d_{\mathrm{Buf}}=1.3 \AA$. b) Anticorrelation of the bonding-antibonding states gap with the distance between the group-III atom buffer layer and the $\mathrm{SiC}$ surface, shown for both substrate terminations (Si- and C-faces).

potential term (Semenoff mass). This is known to be detrimental to the QSH phase, as it tends to compete with the second-nearest neighbor SOC $\underline{3}$.

\section{B. Group-III buffered SiC + stanene}

We now analyze the behavior of stanene, in particular its geometrical and its topological properties, when deposited on the group-III buffer layer. For the sake of clarity, we derive most of the following conclusion by focusing on the specific case of the Al-buffer on SiC. The main purpose is to understand the physical mechanisms behind the interaction between stanene and the buffered substrate. With this in mind, we monitor the evolution of the electronic band structure of stanene as its bonding distance is artificially tuned from an unrealistically large value down to the equilibrium one $d_{\mathrm{eq}} \sim 2.9 \AA$ (see Fig. 4 a). The $2 \times 2$ structure reconstruction induced by the lattice commensuration leads to band foldings 50 . When the stanene-substrate distance is large enough to reduce the hybridization effects and the Coulomb interaction, we recover the freestanding stanene electronic properties. By unfolding the stanene band structure from the $2 \times 2$ Brillouin zone into the primitive $1 \times 1$, we can directly compare with the results of the freestanding description [8]. The reduction of the distance towards the equilibrium geometry and the concomitant interaction causes the opening of hybridization gaps in the stanene band structure. Along with it, we find a distribution (spread) of stanene character over the $\mathrm{Al} / \mathrm{SiC}$ electronic states. When the distance becomes smaller than the critical value $d_{\text {cr }} \sim 3.2 \AA$, we observe a switching of the $\mathbb{Z}_{2}$
TABLE I: Summary of the DFT results obtained for buffer layers made of group III elements (for a geometry of stanene with preserved hexagonal symmetry). Si and $\mathrm{C}$ refer to the silicon and carbon terminated $\mathrm{SiC}$, respectively. $\Delta \mathrm{E}_{\mathrm{K}}$ is the stanene energy gap at the $\mathrm{K}$ point. The dash symbol indicates that the system is metallic. $d_{\mathrm{Buf}}, d$ and $\delta$ are the buffer layer$\mathrm{SiC}$ distance, the stanene-buffer distance and the buckling height, respectively. $\lambda_{v}$ and $3 \sqrt{3} \lambda_{\text {SO }}$ are the Semenoff mass and the effective SOC entering the Hamiltonian at the $\mathrm{K}$ point as given in Eq. 3, respectively. We also report the values of the $\mathbb{Z}_{2}$-invariant, even though in the case of group III this is always 0 at $d=d_{\text {eq }}$. The calculations are performed within GGA-PBE [39]. In the trivial $\mathbb{Z}_{2}=0$ phase, $\Delta \mathrm{E}_{\mathrm{K}}=2\left[\lambda_{v}-\right.$ $\left.3 \sqrt{3} \lambda_{\mathrm{SO}}\right]$.

\begin{tabular}{|c|c|c|c|c|c|}
\hline & & $\mathrm{Al}$ & $\mathrm{Ga}$ & In & $\mathrm{Tl}$ \\
\hline \multicolumn{6}{|c|}{$\mathrm{Si}$} \\
\hline & $\Delta \mathrm{E}_{\mathrm{K}}(\mathrm{meV})$ & 56 & 66 & 60 & 63 \\
\hline & $d_{\mathrm{Buf}}(\AA)$ & 1.7 & 1.8 & 2.0 & 2.2 \\
\hline & $d(\AA)$ & 2.9 & 2.9 & 3.0 & 3.1 \\
\hline & $\delta(\AA)$ & 0.44 & 0.48 & 0.48 & 0.48 \\
\hline & $\mathbb{Z}_{2}$ & 0 & 0 & 0 & 0 \\
\hline & $\lambda_{v}(\mathrm{meV})$ & 49 & 58 & 50 & 52 \\
\hline & $3 \sqrt{3} \lambda_{\mathrm{SO}}(\mathrm{meV})$ & 21 & 25 & 20 & 21 \\
\hline \multicolumn{6}{|c|}{$\mathrm{C}$} \\
\hline & $\Delta \mathrm{E}_{\mathrm{K}}(\mathrm{meV})$ & - & - & - & - \\
\hline & $d_{\mathrm{Buf}}(\AA)$ & 1.3 & 1.4 & 1.8 & 2.1 \\
\hline & $d(\AA)$ & 2.8 & 2.8 & 3.0 & 3.1 \\
\hline & $\delta(\AA)$ & 0.44 & 0.48 & 0.48 & 0.48 \\
\hline & $\mathbb{Z}_{2}$ & - & - & - & - \\
\hline
\end{tabular}


invariant from 1 to 0 (Fig. 4b). This evidence marks the transition from freestanding-like topologically nontrivial to topologically trivial stanene, at least within GGA framework. As we will see in the following, the same mechanism leads to a different outcome in the case of group V.

A change in the topological invariant can only occur through an inversion of the bulk gap [1, 2]. This evolution is indeed what we observe and show in Fig. 4k. First of all, the breaking of the inversion symmetry induced by the presence of the substrate removes the band degeneracies at the K points. This effect goes under the name of valley-contrasting physics, or valleytronics, and is a wellknown property of gated graphene and transition metal dichalcogenides [56, 57. The gap closure is accompanied by a change in the sublattice character of the bands. In fact, in the topological (trivial) phase, the contribution from the bottom Sn (top Sn) atom dominates the valence band maximum, and vice-versa for the conduction band minimum. The closure of the gap occurs through a linear Dirac-like band touching where the sublattice character is equally mixed, as highlighted by the brownish color of the linear branches in the middle panel of Fig. 4p).

In the next section, we will establish an analogy with the topological transition as described by the Kane and Mele model [3], and analyze the quantitative role of the Semenoff mass. Note that the trend observed here for stanene on $\mathrm{Al} / \mathrm{SiC}$ is common to all the group-III buffer setups, regardless of the $\mathrm{SiC}$ termination (see Table I). As shown in Fig. 3b, it holds that the heavier the buffer atom deposited on $\mathrm{SiC}$, the smaller the bulk gap. For this reason, the most favorable group-III buffer for growing quasi-freestanding stanene is Al. The latter leads, however, to a topologically trivial configuration for stanene, demonstrating how going downwards in the "triels" group of the periodic table is not a promising approach to obtain $\mathbb{Z}_{2}=1$.

\section{ROLE OF THE SEMENOFF MASS}

Interpreted within the framework of the Kane and Mele model [3], our tight-binding Hamiltonian reads

$$
\begin{aligned}
H= & t \sum_{\langle i j\rangle, \alpha} c_{i, \alpha}^{\dagger} c_{j, \alpha}+i \lambda_{\mathrm{SO}} \sum_{\langle\langle i j\rangle\rangle, \alpha \alpha^{\prime}} \nu_{i j} c_{i, \alpha}^{\dagger} s_{\alpha \alpha^{\prime}}^{z} c_{j, \alpha^{\prime}} \\
& +i \lambda_{R} \sum_{\langle i j\rangle, \alpha \alpha^{\prime}} c_{i, \alpha}^{\dagger}\left(\mathbf{s} \times \hat{\mathbf{d}}_{i j}\right)_{\alpha \alpha^{\prime}}^{z} c_{j, \alpha^{\prime}} \\
& +\lambda_{v} \sum_{i, \alpha} \xi_{i} c_{i, \alpha}^{\dagger} c_{i, \alpha}
\end{aligned}
$$

where the first is a nearest neighbor hopping term on the honeycomb lattice and the second a mirror symmetric SOC one (here $\nu_{i j} \hat{\mathbf{u}}_{z}=(2 / \sqrt{3})\left(\hat{\mathbf{d}}_{1} \times \hat{\mathbf{d}}_{2}\right)$ where $\hat{\mathbf{d}}_{1,2}$ are unit vectors along the two bonds from site $j$ to site $i$, and $\hat{\mathbf{u}}_{z}$ is a unit vector perpendicular to the stanene plane), and $\mathbf{s}$ are the Pauli matrices for the electron spin. The third term is a nearest-neighbor Rashba coupling due to a perpendicular electric field or to an interaction with a substrate and the last term sets the staggered potential (Semenoff mass) $\lambda_{v}\left(\xi_{i}= \pm 1\right.$ depending on the sublattice). The latter differentiates the on-site energies of the two atoms constituting the bipartite honeycomb lattice. Without the Rashba term, the momentum-space Hamiltonian assumes the form

$$
\begin{aligned}
H(\mathbf{k}) & =t(1+2 \cos x \cos y) \Gamma_{1}-2 t \cos x \sin y \Gamma^{12} \\
& +\lambda_{\mathrm{SO}}(2 \sin 2 x-4 \sin x \cos y) \Gamma^{15}+\lambda_{v} \Gamma_{2}
\end{aligned}
$$

with $x=k_{x} a / 2$ and $y=\sqrt{3} k_{y} a / 2$ ( $a$ being the hexagonal unit cell lattice constant, i.e. $\overline{\langle i j\rangle}=a / \sqrt{3}), \Gamma_{1}=\sigma_{x} \otimes s_{0}$, $\Gamma_{2}=\sigma_{z} \otimes s_{0}$ and $\Gamma_{5}=\sigma_{y} \otimes s_{z}$ three of the five Dirac matrices with $\sigma_{i}$ describing the sublattice degree of freedom and $\Gamma^{a b}=\left[\Gamma_{a}, \Gamma_{b}\right] / 2 i$. At the $\mathrm{K}$ point, the Hamiltonian is diagonal in the sublattice basis $A \uparrow, B \uparrow, A \downarrow, B \downarrow$ :

$$
\left[\begin{array}{cccc}
\lambda_{v}-3 \sqrt{3} \lambda_{\mathrm{SO}} & 0 & 0 & 0 \\
0 & -\left(\lambda_{v}-3 \sqrt{3} \lambda_{\mathrm{SO}}\right) & 0 & 0 \\
0 & 0 & \lambda_{v}+3 \sqrt{3} \lambda_{\mathrm{SO}} & 0 \\
0 & 0 & 0 & -\left(\lambda_{v}+3 \sqrt{3} \lambda_{\mathrm{SO}}\right)
\end{array}\right]
$$

The two $\uparrow$ - and the two $\downarrow$-eigenvalues with smaller and larger splitting, respectively, give perfect account of the four bands visible in the actual DFT calculation (Fig. 4c). The matching between the DFT result and the Kane-Mele model can be pushed further upon comparing the sublattice character of these four eigenvalues and its evolution with $d$. We define $d_{\text {cr }}$ as the distance at which the two lowest-lying DFT eigenvalues touch each other. For $d<d_{\text {cr }}$ the character of both the uppermost eigenvalues is on one sublattice, namely the one corresponding to the bottom Sn-atom, while both eigenvalues in valence band belong to the top Sn. For $d>d_{\text {cr }}$ the sublattice character at the $\mathrm{K}$ point is instead interchanged, a situation which in the mapping onto the Kane-Mele is obtained in the QSH phase for values of the Semenoff mass $\lambda_{v}<3 \sqrt{3} \lambda_{\mathrm{SO}}[3$. In the topological phase with inverted sublattice character, the lattice site with the highest on-site energy $\left(+\lambda_{v}\right)$ contributes to the valence band maximum at $\mathrm{K}$, at odds with the trivial atomic limit.

Let us note that the inversion of sublattice character is well defined only at the $K$ and $\mathrm{K}^{\prime}$ points, where the Kane-Mele Hamiltonian is diagonal in the original basis. A suitable definition of the character over the whole Brillouin zone can be obtained by rotating the Kane-Mele Hamiltonian at each $k$-point in the basis of the eigenvectors of (2) calculated at $\lambda_{\mathrm{SO}}=0$. At the $\Gamma$ point, for instance, the Kane-Mele model is made of two identical $2 \times 2$ blocks, with $\pm \lambda_{v}$ on the diagonal and $3 t$ between the $A$ and $B$ sites. Going away from the $\Gamma$ point and continuing to use the "bonding"- "antibonding" basis defined above, the SOC acquires off-diagonal elements which mix the "bonding"- "antibonding" eigenvectors approaching $\mathrm{K}$ and $\mathrm{K}^{\prime}$ leading to the inversion at the topological phase transition.

So far we have discussed the qualitative correspondence between the Kane-Mele model and the DFT results. Now, we make a more quantitative comparison 
a)

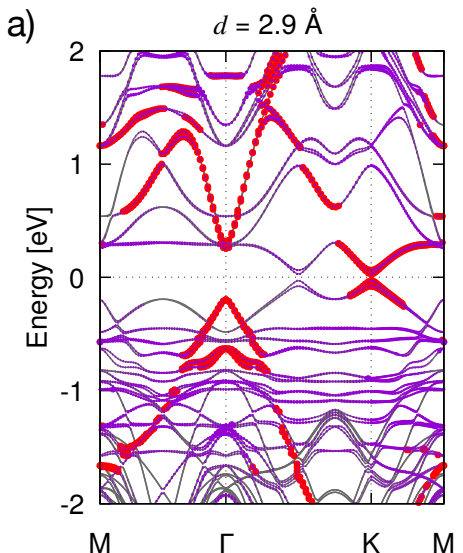

b)

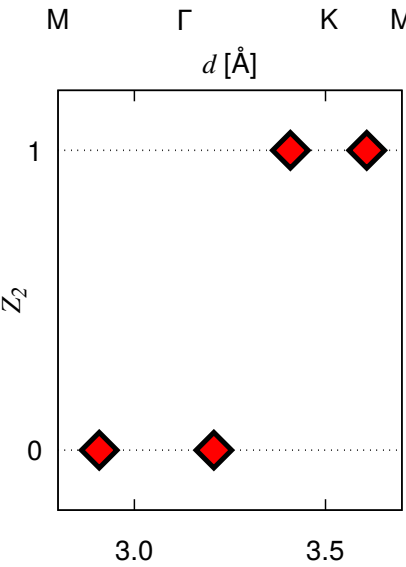

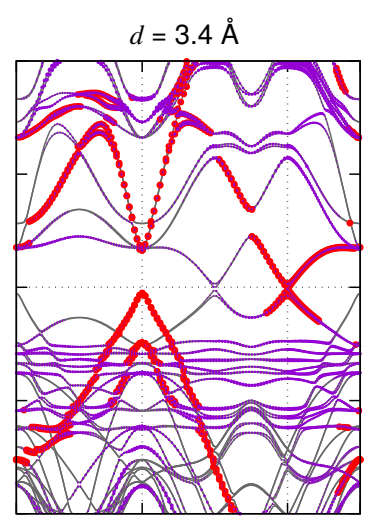

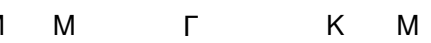

C)

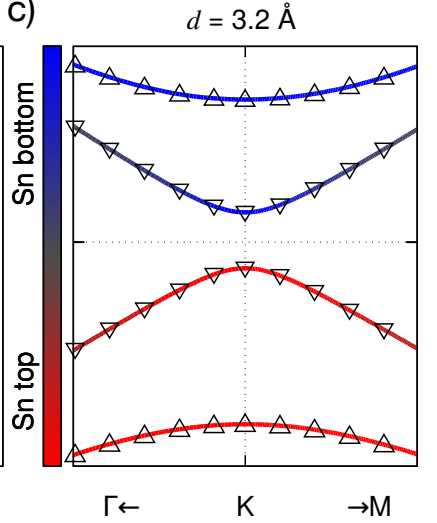

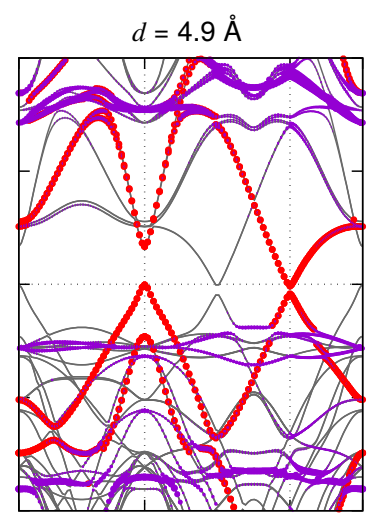

$\mathrm{M}$
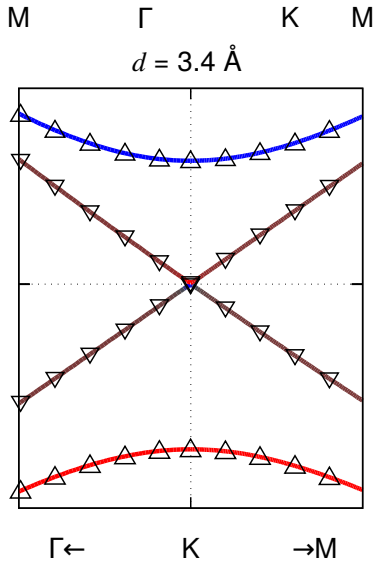

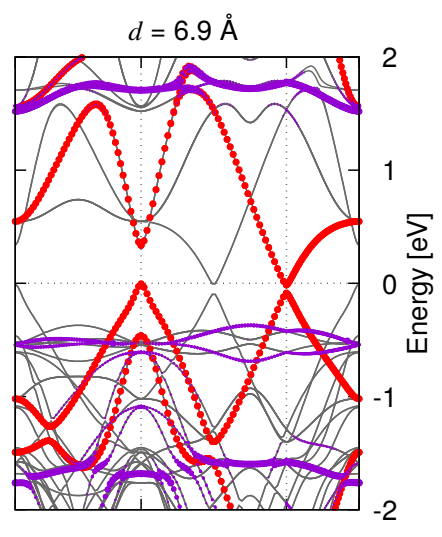

$\mathrm{M} \quad \Gamma \quad \mathrm{K} \quad \mathrm{M}$ $d=3.6 \AA$

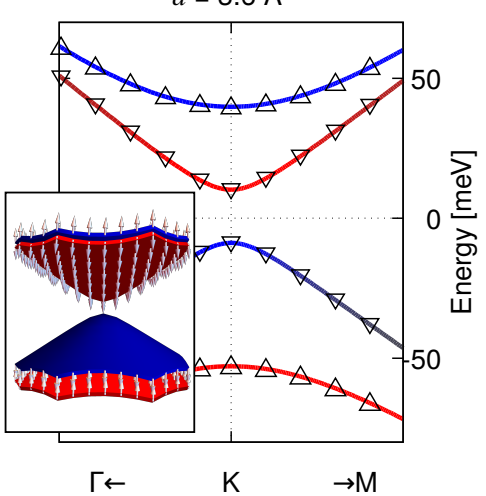

FIG. 4: a) Evolution of the stanene/Al/SiC electronic band structure at different stanene-Al buffer distances $(d=2.9 \AA, 3.4 \AA$, $4.9 \AA$ and $6.9 \AA$ from left to right panels). Red dots highlight the unfolding weight from the $2 \times 2$ Brillouin zone onto the primitive $1 \times 1$, while the violet color code refers to the orbital contribution from the buffer atoms. b) Behaviour of the $\mathbb{Z}_{2}$ topological invariant close to the critical transition distance $d_{\mathrm{cr}}$. c) Zooms around the $\mathrm{K}$ point across the topological transition. Red and blue colors refer to orbital character from top and bottom Sn atoms, respectively. In the inset we report a three-dimensional view of the spin-texture. The in-plane $S_{x}$ and $S_{y}$ components are negligible compared to the out-of-plane $S_{z}$ component.

by estimating the Semenoff mass and SOC term via a fit of the DFT eigenvalues at the K point with the analytic expressions given in Eq. 3. For the $\mathrm{Al}$ buffer, at $d_{\text {eq }}$, we extract a difference between the $p_{z}$ onsite energies of the top- and bottom-Sn atoms of $2 \lambda_{v}=0.098$ $\mathrm{eV}$. The highest levels belong to the bottom Sn-atoms, that feel the buffer layer stronger than the top ones and are hence pushed up in energy. In the Kane-Mele Hamiltonian $\lambda_{\text {SO }}$ represents a non-local term which accounts for the coupling of orbitals with parallel spin on the same sublattice. However, a finite buckling angle introduces a non-vanishing local contribution to the SOC [58. We estimate that, in stanene on $\mathrm{Al} / \mathrm{SiC}, 3 \sqrt{3} \lambda_{\mathrm{SO}} \sim 21$ $\mathrm{meV}$. Therefore, the relationship $\lambda_{v}>3 \sqrt{3} \lambda_{\text {SO }}$ holds for stanene on the $\mathrm{Al}$ buffer at the equilibrium distance, confirming the trivial ground state found in our DFT computations. As can be inferred from Table 1. the same relationship holds for all the investigated group-III buffers.

Let us briefly comment on the quantitative estimate of the Semenoff mass: an alternative way of extracting the staggered potential is from the difference of the $p_{z}$-local levels of the two sublattices via a Wannier projection of the DFT band-structure. In order to get a satisfactory agreement with DFT, the projection must contain all the $p$-orbitals of tin as well as orbitals from the buffer atoms. This means that the resulting Wannier Hamiltonian is defined on a much larger Hilbert space than the one of the Kane-Mele model, which assumes one single orbital per site. To directly connect to $\lambda_{v}$, one would have to further downfold the Wannier Hamiltonian onto a $p_{z^{-}}$ type low-energy model. A direct projection onto such a minimal model, however, turns out to yield a poor correspondence with the DFT bands. Furthermore, there is another argument in favor of an extended local basis, namely that this allows to disentangle hybridization effects stemming from the buffer. As described above, we adopt the simpler procedure based on the fit of the DFT eigenvalues at the $\mathrm{K}$ point to the analytic results of Eq. 3 .

In the previous analysis, we have neglected the Rashba coupling in Eq. 1. The presence of a finite $\lambda_{R}$ mixes the $\uparrow$ - and $\downarrow$-blocks of the Hamiltonian breaking the conservation of the $z$-component of the spin. Moreover, a chiral spin-texture appears around the $\mathrm{K}$ point. We checked that this effect is negligible, as shown in the inset to 
a)

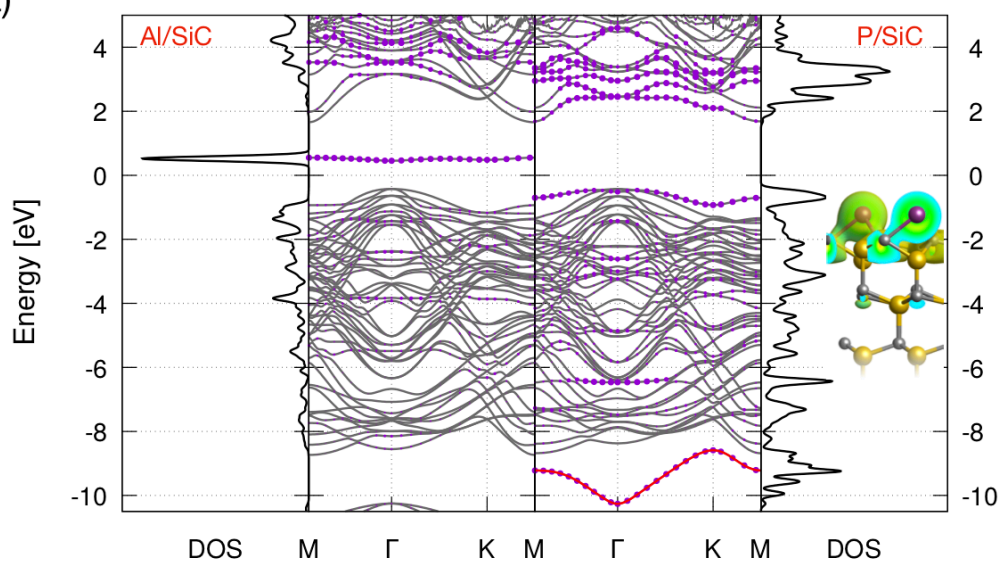

c)

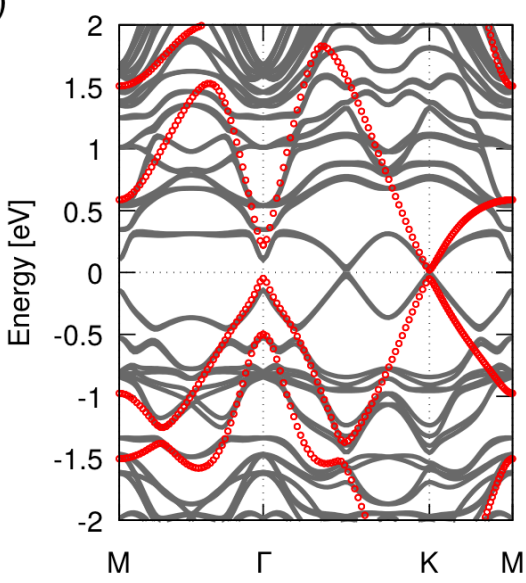

d)

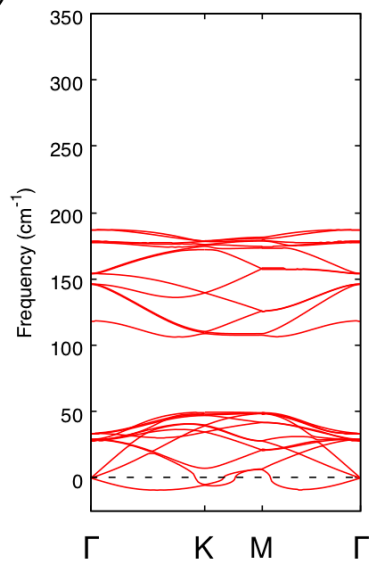

b)

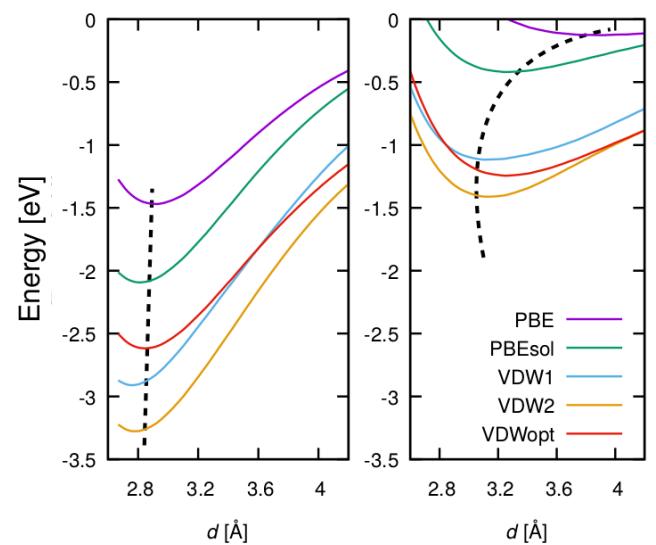

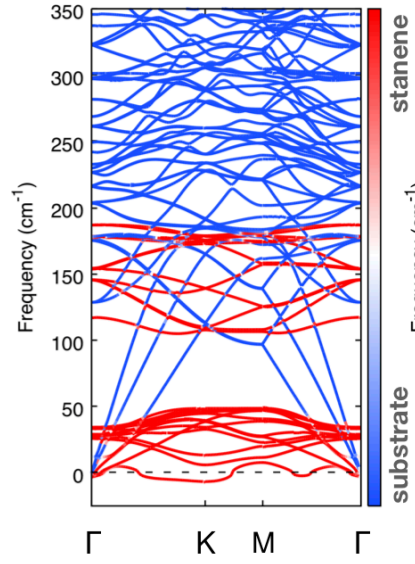

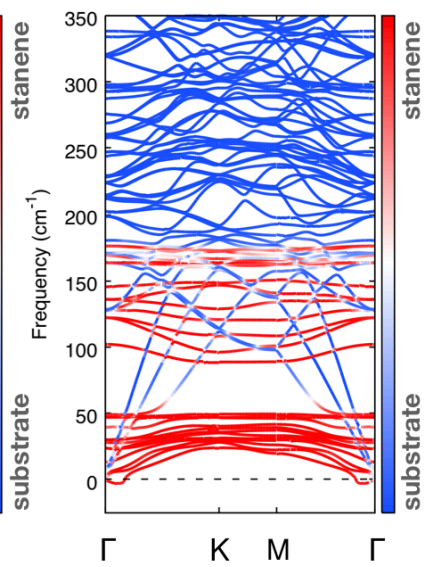

FIG. 5: a) Buffer resolved density of the states and band structure of $\mathrm{Al} / \mathrm{SiC}$ and $\mathrm{P} / \mathrm{SiC}$ (C-face hereafter in this caption), respectively. The dots in the band structure highlight the orbital weight of buffer atoms. In the inset, the charge distribution projected on the states forming the lone pair around $9 \mathrm{eV}$ of binding energy for $\mathrm{P} / \mathrm{SiC}$ is shown. b) Binding energy curves for stanene on $\mathrm{Al}$ Si-terminated $\mathrm{SiC}$ (left panel) and on P C-terminated $\mathrm{SiC}$ (right panel) with and without different flavours of van der Waals corrected functionals (PBE [39, PBEsol [40, VDW1 [1], VDW2 [42, VDWopt 43]). The black dashed lines are guide to the eye to track the evolution of the equilibrium distance within the tested approximations. c) band structure (with VDW1 van der Waals correction) of $2 \times 2$ stanene on $\mathrm{P} / \mathrm{SiC}$ with the red dots referring to freestanding stanene in the primitive $1 \times 1$ structure. d) Phonon spectra of freestanding stanene (leftmost panel) and stanene on $\mathrm{P} / \mathrm{SiC}$ at the $3.9 \AA \mathrm{PBE}$

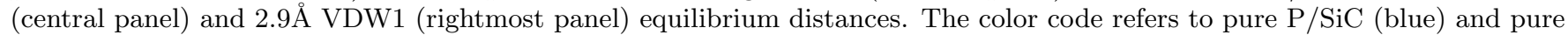
stanene (red) contributions to the phonon modes.

Fig. 4k, proving that our assumption $\lambda_{R}=0$ is justified and does not affect the qualitative arguments given above. Let us additionally note that, as recently demonstrated, the impact of the Rashba coupling in 2D superstructures can be reduced by a proper choice of the lattice commensuration [59].

\section{STANENE ON GROUP-V BUFFER}

Equipped with the microscopic understanding gained in the previous section of the mechanism responsible for the topological transition in stanene on the buffer layer, we shall now address the key question of our study: how to realize quasi freestanding topologically non-trivial stanene. From our analysis of group III, we have learned that the buffer in-gap states have to stay as far as possible away from $E_{F}$ and, at the same time, the Semenoff mass term must be minimized. To limit the detrimental effect of these antibonding states, the corresponding bonds ought to be saturated. Two more valence electrons in buffer exactly serve this purpose, offered by the $p$-shell of group- $\mathrm{V}$ atoms.

In Fig. 5 we compare the density of the states (DOS) and band structure of $\mathrm{Al} / \mathrm{SiC}$ and $\mathrm{P} / \mathrm{SiC}$ (C-face). From the comparison between group-III and $\mathrm{V}$ buffer it is clear that our expectation of the quenching of the antibonding state is fulfilled: for group $\mathrm{V}$ we indeed observe a chemically inert lone pair characterized by a pronounced $s$-type orbital character (see inset in Fig. 5a). This is located 
around $10 \mathrm{eV}$ below the Fermi level, leaving as a consequence more "room" within the gap for an interferencefree positioning of the stanene layer. Thanks to the reduced monolayer/substrate interaction, the induced Semenoff mass is indeed smaller. This is illustrated in Table II. where we summarize our results for stanene on group$\mathrm{V}$ buffer/SiC substrates. All the calculated values of $\lambda_{v}$ are sensibly reduced compared to the ones reported in Table I. Furthermore, contrary to group III, in the case of group $\mathrm{V}$ buffers the hexagonal symmetry of stanene is preserved during the relaxation, reflecting the much weaker hybridization strength.

Before moving to the analysis of the resulting electronic properties, we turn our attention to the nature of the bonding between stanene and the buffer layer. To this purpose, in Fig. 5b we show the binding energies of stanene on $\mathrm{Al}$ (left panel) and $\mathrm{P}$ (right panel) buffer/SiC within different levels of sophistication, explicitly including long-range dispersion to account for van der Waals interactions. The difference in the two behaviour suggests a distinct nature of the bonding. While on group III buffer/SiC van der Waals corrections do not change qualitatively the equilibrium geometry of stanene, a sizeable reduction of the equilibrium distance occurs for group $\mathrm{V}$ buffer/SiC. Such an evidence hints at a strong covalent nature of the bonding on group-III buffer, as a result of the hybridization between stanene and the antibonding in-gap state. On the other hand, the inert lone pair prevents from any form of chemical bonding, leaving room for the predominance of the van der Waals interaction.

TABLE II: Same as Table II but for buffer layers made of group-V elements. The column on the left refers to Si-face or C-face of the $\mathrm{SiC}$ substrate. The asterisk $(*)$ in the entries of $\delta$ indicates that the stanene low-buckled hexagonal geometry is not preserved anymore and it is not possible to define a buckling parameter. The calculations are performed within the VDW1 framework [41. In the topological $\mathbb{Z}_{2}=1$ phase, $\Delta \mathrm{E}_{\mathrm{K}}=2\left[3 \sqrt{3} \lambda_{\mathrm{SO}}-\lambda_{v}\right]$.

\begin{tabular}{|c|c|c|c|c|c|}
\hline & & $\mathrm{P}$ & As & $\mathrm{Sb}$ & $\mathrm{Bi}$ \\
\hline \multicolumn{6}{|c|}{$\overline{\mathrm{Si}}$} \\
\hline & $\Delta \mathrm{E}_{\mathrm{K}}(\mathrm{meV})$ & - & - & - & - \\
\hline & $d_{\mathrm{Buf}}(\AA)$ & 1.8 & 2.0 & 2.1 & 2.2 \\
\hline & $d(\AA)$ & 1.9 & 1.7 & 3.0 & 3.2 \\
\hline & $\delta(\AA)$ & $*$ & $*$ & 0.40 & 0.43 \\
\hline & $\mathbb{Z}_{2}$ & - & - & - & - \\
\hline \multicolumn{6}{|c|}{$\bar{C}$} \\
\hline & $\Delta \mathrm{E}_{\mathrm{K}}(\mathrm{meV})$ & 13 & 34 & 1 & 13 \\
\hline & $d_{\mathrm{Buf}}(\AA)$ & 1.2 & 1.4 & 1.6 & 1.8 \\
\hline & $d(\AA)$ & 2.9 & 3.0 & 3.2 & 3.2 \\
\hline & $\delta(\AA)$ & 0.43 & 0.42 & 0.42 & 0.42 \\
\hline & $\mathbb{Z}_{2}$ & 1 & 1 & 1 & 1 \\
\hline & $\lambda_{v}(\mathrm{meV})$ & 16 & 6 & 19 & 10 \\
\hline & $3 \sqrt{3} \lambda_{\mathrm{SO}}(\mathrm{meV})$ & 23 & 23 & 19 & 17 \\
\hline
\end{tabular}

The band structure of stanene on C-terminated $\mathrm{P} / \mathrm{SiC}$ at the van der Waals equilibrium geometry is shown in Fig. 5r. The Dirac physics around the $\mathrm{K}$ point resembles to a large extent the one of freestanding stanene [8]. Our conclusion is further substantiated by the explicit computation of the $\mathbb{Z}_{2}$ invariant (not shown), where a Kramers' pair switching indicates the non-trivial topological nature $\left(\mathbb{Z}_{2}=1\right)$ [6].

Our calculations reveal that $\mathrm{P}$ and As buffer-atoms on C-terminated $\mathrm{SiC}$ are ideal templates to host stanene in the QSH phase. The high electronegativity of carbon plays an important role. The resulting strengthening of the bonding of the buffer atoms to the substrate, and in turn of the hybridization between the in-gap bands and the continuum of $\mathrm{SiC}$ electronic states, sets favourable conditions for a topological ground state. We predict stanene to be a QSH on $\mathrm{Bi}$ as well, but it is likely that $\mathrm{Bi}$ on $\mathrm{SiC}$ prefers the $2 / 3$ coverage, as recently reported in the case of bismuthene/SiC [12]. Stanene on $\mathrm{Sb} / \mathrm{SiC}$ is right at the verge of the topological transition, being the gap at the $\mathrm{K}$ point not larger than $\sim 1 \mathrm{meV}$. The configurations on the other termination of $\mathrm{SiC}$ are instead metallic and in the cases of $\mathrm{P}$ and As, also highly distorted. Being less electronegative than C, Si attracts less negative charge from the buffer atoms compared to what $\mathrm{C}$ does, resulting in turn in a larger $\mathrm{SiC} /$ buffer distance $d_{\text {Buf }}$ (see Table II). This confirms that the C-face, in combination with group- $\mathrm{V}$ buffer layers, offers the ideal degree of hybridization to host stanene without spoiling the QSH phase.

Having assessed the possibility to achieve a topological ground state for stanene, an important issue is yet to be addressed. It is known that freestanding stanene as predicted in Ref. 8 is not dynamically stable in the low-buckled (LB) geometry of interest here [60]. This instability is a consequence of negative phonon frequencies that the flexural $\mathrm{ZA}$ mode has along the $\Gamma-K$ and $\Gamma-\mathrm{M}$ directions, as shown in the leftmost panel of Fig. 5d). The origin of negative frequencies is ascribed to the weak $\pi-\pi$ bonding of LB stanene that fails to stabilize a buckled configuration. It is interesting to note how a dumbbell (DB) stanene configurations has been theoretically proposed to be stable and topologically non-trivial on a variety of different substrates [60]. At odds with LB stanene, however, in DB stanene the band inversion induced by the SOC occurs at the $\Gamma$ point, and the Diraclike physics at the $\mathrm{K}$ point is irredeemably spoiled.

The substrate engineering based on our buffer strategy turns out to be decisive to stabilize stanene in the LB configuration. As shown in the central and rightmost panels of Fig. 5d), the interaction with the substrate reduces the negative phonon frequencies, eventually ending up in a stable configuration at the van der Waals equilibrium distance. In such a configuration, all the frequencies of phonon modes associated to stanene are positive. The small still imaginary frequencies in the long-wavelength $\mathbf{q} \rightarrow 0$ flexural acoustic ZA mode is a well known artifact that is not related to a structural instability 61,63 . 
The stabilization of the ZA phonon mode via interaction with the buffer is a result of the very nature of this mode, which in atomically thin systems involves a vertical bending of the layer, i.e. a flexural mode in the direction of the substrate. We cannot exclude at this point a highbuckled (HB) configuration of stanene, since it has been reported that for freestanding tin and lead monolayers, a metallic HB phase is energetically favourable against the LB one 64. However, we stress again here that the role of the substrate, within the concept we propose in this work, is pivotal to accomplish a quasi-freestanding LB phase, which is dynamically stable but still retains a QSH physics at the K point.

\section{CONCLUSIONS}

Through a systematic first-principles analysis we have unveiled the mechanisms underlying the delicate QSH formation in stanene grown on a $\mathrm{SiC}(0001)$ substrate, and how these growth conditions can be fundamentally improved by an appropriate buffer between substrate and monolayer. We followed the guiding principle that a technologically relevant substrate is crucial for any future integration of QSH systems in novel spin-based devices. $\mathrm{SiC}$ distinguishes itself as one of the most promising substrate candidates, we have accomplished a substrate engineering mechanism that employs a buffer layer for the saturation of the substrate dangling bonds. We have further revealed the microscopic processes that are deleterious to the formation of the topological phase, owing to the electronic hybridization between the substrate and the stanene monolayer. Combining these insights, we predict a stable QSH phase of stanene in the four cases of group-V buffered SiC.

By analyzing the hybridization between the stanene layer and the buffer, and by mapping the full ab-initio Hamiltonian onto a generalized effective Hamitonian including a staggered potential (Semenoff mass term), we show that some buffer layers are more suitable than others to protect the QSH formation in the monolayer. The strategy we follow then is to minimize the detrimental staggered potential by choosing such atoms which have their bonding and antibonding states energetically far away from the chemical potential. This leads us to concrete suggestions for promising buffer materials, in particular the use of group-V elements ( $\mathrm{P}$ and $\mathrm{As}$ ).

Note that our theoretical study only establishes a lower bound to the potential range of parameter space in which the QSH phase in stanene might be stabilized by proper substrate engineering. This is because our DFT approach utilizes the GGA approximation, which, due to the prohibitive size of the reconstructions we considered, would be extremely time-consuming to replace it by alternative procedures. The use of GGA suggests that a $\sim 20 \%$ underestimation of the antibonding states energy position is possible (see Fig. 3a), and that some of the trivial configurations we have found (Table $\mathrm{I}$ and II ) are in fact still in the QSH domain. In terms of an explicit analysis of the buffer-assisted growth of stanene on $\mathrm{SiC}$, we reported the first detailed LEED pattern (Fig. 2) demonstrating a $\sqrt{3} \times \sqrt{3} R\left(30^{\circ}\right)$ reconstruction of $\mathrm{SiC}$ after $\mathrm{Al}$ deposition. Together, our efforts constitute promising steps towards the accomplishment for the realization of quantum spin Hall candidate materials through buffer engineering.

\section{Acknowledgement}

The authors acknowledge G. Profeta for fruitful and inspiring discussions. This work was supported by the DFG through SFB1170 "ToCoTronics" and by ERCStG-336012-Thomale-TOPOLECTRICS. We gratefully acknowledge the Gauss Centre for Supercomputing e.V. (www.gauss-centre.eu) for funding this project by providing computing time on the GCS Supercomputer SuperMUC at Leibniz Supercomputing Centre (www.lrz.de).
[1] M. Z. Hasan and C. L. Kane, Colloquium: Topological insulators, Rev. Mod. Phys. 82, 3045 (2010).

[2] X.-L. Qi and S.-C. Zhang, Topological insulators and superconductors, Rev. Mod. Phys. 83, 1057 (2011).

[3] C. L. Kane and E. J. Mele, $Z_{2}$, Phys. Rev. Lett. 95, 146802 (2005).

[4] C. L. Kane and E. J. Mele, Quantum Spin Hall Effect in Graphene, Phys. Rev. Lett. 95, 226801 (2005).

[5] B. A. Bernevig, T. L. Hughes, and S.-C. Zhang, Quantum Spin Hall Effect and Topological Phase Transition in HgTe Quantum Wells, Science 314, 1757 (2006).

[6] M. König, S. Wiedmann, C. Brüne, A. Roth, H. Buhmann, L. W. Molenkamp, X.-L. Qi, and S.-C. Zhang, Quantum Spin Hall Insulator State in HgTe Quantum Wells, Science 318, 766 (2007).

[7] A. Molle, J. Goldberger, M. Houssa, Y. Xu, S.-C. Zhang, and D. Akinwande, Buckled two-dimensional
Xene sheets, Nature Materials 16, 163 (2017).

[8] Y. Xu, B. Yan, H.-J. Zhang, J. Wang, G. Xu, P. Tang, W. Duan, and S.-C. Zhang, Large-Gap Quantum Spin Hall Insulators in Tin Films, Phys. Rev. Lett. 111, 136804 (2013).

[9] F. Geissler, J. C. Budich, and B. Trauzettel, Group theoretical and topological analysis of the quantum spin hall effect in silicene, New Journal of Physics 15, 085030 (2013).

[10] L. Zhang, P. Bampoulis, A. N. Rudenko, Q. Yao, A. van Houselt, B. Poelsema, M. I. Katsnelson, and H. J. W. Zandvliet, Structural and Electronic Properties of Germanene on $\mathrm{MoS}_{2}$, Phys. Rev. Lett. 116, 256804 (2016).

[11] F.-F. Zhu, W.-J. Chen, Y. Xu, C.-L. Gao, D.-D. Guan, C.-H. Liu, D. Qian, S.-C. Zhang, and J.-F. Jia, Epitaxial growth of two-dimensional stanene, Nature Materials $\mathbf{1 4}$, 1020 (2015). 
[12] F. Reis, G. Li, L. Dudy, M. Bauernfeind, S. Glass, W. Hanke, R. Thomale, J. Schäfer, and R. Claessen, Bismuthene on a SiC substrate: A candidate for a hightemperature quantum spin Hall material, Science 357, 287 (2017).

[13] X. Qian, J. Liu, L. Fu, and J. Li, Quantum spin Hall effect in two-dimensional transition metal dichalcogenides, Science 346, 1344 (2014).

[14] S. Tang, C. Zhang, D. Wong, Z. Pedramrazi, H.-Z. Tsai, C. Jia, B. Moritz, M. Claassen, H. Ryu, S. Kahn, J. Jiang, H. Yan, M. Hashimoto, D. Lu, R. G. Moore, C.-C. Hwang, C. Hwang, Z. Hussain, Y. Chen, M. M. Ugeda, Z. Liu, X. Xie, T. P. Devereaux, M. F. Crommie, S.-K. Mo, and Z.-X. Shen, Quantum spin Hall state in monolayer $W T e_{2}$, Nat. Phys. 13, 683 (2017).

[15] S. Ok, L. Muechler, D. Di Sante, G. Sangiovanni, R. Thomale, and T. Neupert, Custodial glide symmetry of quantum spin Hall edge modes in $W^{T} e_{2}$ monolayer, arXiv:1811.00551 .

[16] C.-Z. Xu, Y.-H. Chan, P. Chen, X. Wang, D. Flötotto, J. A. Hlevyack, G. Bian, S.-K. Mo, M.-Y. Chou, and T.-C. Chiang, Gapped electronic structure of epitaxial stanene on InSb(111), Phys. Rev. B 97, 035122 (2018).

[17] Z. Ni, E. Minamitani, Y. Ando, and S. Watanabe, Germanene and stanene on two-dimensional substrates: Dirac cone and $Z_{2}$ invariant, Phys. Rev. B 96, 075427 (2017).

[18] F. Matusalem, F. Bechstedt, M. Marques, and L. K. Teles, Quantum spin Hall phase in stanene-derived overlayers on passivated SiC substrates, Phys. Rev. B 94, 241403 (2016).

[19] F. Matusalem, D. S. Koda, F. Bechstedt, M. Marques, and L. K. Teles, Deposition of topological silicene, germanene and stanene on graphene-covered SiC substrates, Scientific Reports 7, 15700 (2017).

[20] S. Glass, G. Li, F. Adler, J. Aulbach, A. Fleszar, R. Thomale, W. Hanke, R. Claessen, and J. Schäfer, Triangular Spin-Orbit-Coupled Lattice with Strong Coulomb Correlations: Sn Atoms on a SiC(0001) Substrate, Phys. Rev. Lett. 114, 247602 (2015).

[21] S. Glass, F. Reis, M. Bauernfeind, J. Aulbach, M. R. Scholz, F. Adler, L. Dudy, G. Li, R. Claessen, and J. Schäfer, Atomic-Scale Mapping of Layer-by-Layer Hydrogen Etching and Passivation of SiC(0001) Substrates, The Journal of Physical Chemistry C 120, 10361 (2016).

[22] M. Houssa, G. Pourtois, V. V., Afanasév, and A. Stesmans, Can silicon behave like graphene? a first-principles study, Applied Physics Letters 97, 112106 (2010).

[23] M. Kanno, R. Arafune, C. L. Lin, E. Minamitani, M. Kawai, and N. Takagi, Electronic decoupling by $h-B N$ layer between silicene and Cu(111): A dft-based analysis, New Journal of Physics 16, 105019 (2014).

[24] F. d'Acapito, S. Torrengo, E. Xenogiannopoulou, P. Tsipas, J. M. Velasco, D. Tsoutsou, and A. Dimoulas, Evidence for Germanene growth on epitaxial hexagonal $(h)$ AlN on $A g(111)$, Journal of Physics: Condensed Matter 28, 045002 (2016).

[25] Z. Ni, Q. Liu, K. Tang, J. Zheng, J. Zhou, R. Qin, Z. Gao, $\mathrm{D}$. Yu, and $\mathrm{J} . \mathrm{Lu}$, Tunable bandgap in silicene and germanene, Nano Letters 12, 113 (2012).

[26] Y. Du, J. Zhuang, J. Wang, Z. Li, H. Liu, J. Zhao, X. Xu, H. Feng, L. Chen, K. Wu, X. Wang, and S. X. Dou, Quasi-freestanding epitaxial silicene on $\mathrm{Ag}(111)$ by oxygen intercalation, Science Advances 2 (2016).
[27] T. P. Kaloni and U. Schwingenschlögl, Weak interaction between germanene and GaAs(0001) by h intercalation: A route to exfoliation, Journal of Applied Physics 114, 184307 (2013).

[28] C. Virojanadara, S. Watcharinyanon, A. A. Zakharov, and L. I. Johansson, Epitaxial graphene on $6 \mathrm{H}-\mathrm{SiC}$ and Li intercalation, Phys. Rev. B 82, 205402 (2010).

[29] C. Enderlein, Y. S. Kim, A. Bostwick, E. Rotenberg, and $\mathrm{K}$. Horn, The formation of an energy gap in graphene on ruthenium by controlling the interface, New Journal of Physics 12, 033014 (2010).

[30] S. Oida, F. R. McFeely, J. B. Hannon, R. M. Tromp, M. Copel, Z. Chen, Y. Sun, D. B. Farmer, and J. Yurkas, Decoupling graphene from SiC(0001) via oxidation, Phys. Rev. B 82, 041411 (2010).

[31] F. Varchon, R. Feng, J. Hass, X. Li, B. N. Nguyen, C. Naud, P. Mallet, J.-Y. Veuillen, C. Berger, E. H. Conrad, and L. Magaud, Electronic structure of epitaxial graphene layers on SiC: Effect of the substrate, Phys. Rev. Lett. 99, 126805 (2007).

[32] S. Watcharinyanon, C. Virojanadara, J. Osiecki, A. Zakharov, R. Yakimova, R. Uhrberg, and L. Johansson, Hydrogen intercalation of graphene grown on $6 \mathrm{H}-$ SiC(0001), Surface Science 605, 1662 (2011).

[33] K. V. Emtsev, A. Bostwick, K. Horn, J. Jobst, G. L. Kellogg, L. Ley, J. L. McChesney, T. Ohta, S. A. Reshanov, J. Röhrl, E. Rotenberg, A. K. Schmid, D. Waldmann, H. B. Weber, and T. Seyller, Towards wafer-size graphene layers by atmospheric pressure graphitization of silicon carbide, Nature Materials 8, 203 (2009).

[34] S. Y. Zhou, G.-H. Gweon, A. V. Fedorov, P. N. First, W. A. de Heer, D.-H. Lee, F. Guinea, A. H. Castro Neto, and A. Lanzara, Substrate-induced bandgap opening in epitaxial graphene, Nature Materials 6, 770 (2007).

[35] G. W. Semenoff, Condensed-Matter Simulation of a Three-Dimensional Anomaly, Phys. Rev. Lett. 53, 2449 (1984).

[36] G. Kresse and J. Furthmüller, Efficient iterative schemes for ab initio total-energy calculations using a plane-wave basis set, Phys. Rev. B 54, 11169 (1996).

[37] G. Kresse and D. Joubert, From ultrasoft pseudopotentials to the projector augmented-wave method, Phys. Rev. B 59, 1758 (1999).

[38] P. E. Blöchl, Projector augmented-wave method, Phys. Rev. B 50, 17953 (1994).

[39] J. P. Perdew, K. Burke, and M. Ernzerhof, Generalized Gradient Approximation Made Simple, Phys. Rev. Lett. 77, 3865 (1996).

[40] J. P. Perdew, A. Ruzsinszky, G. I. Csonka, O. A. Vydrov, G. E. Scuseria, L. A. Constantin, X. Zhou, and K. Burke, Restoring the density-gradient expansion for exchange in solids and surfaces, Phys. Rev. Lett. 100, 136406 (2008).

[41] S. Grimme, Semiempirical gga-type density functional constructed with a long-range dispersion correction, J. Comp. Chem. 27, 1787 (2006).

[42] A. Tkatchenko and M. Scheffler, Accurate molecular van der waals interactions from ground-state electron density and free-atom reference data, Phys. Rev. Lett. 102, 073005 (2009).

[43] J. Klimeš, D. R. Bowler, and A. Michaelides, Chemical accuracy for the van der waals density functional, J. Phys.: Cond. Matt. 22, 022201 (2010).

[44] S. Steiner, S. Khmelevskyi, M. Marsmann, and G. Kresse, Calculation of the magnetic anisotropy 
with projected-augmented-wave methodology and the case study of disordered $\mathrm{Fe}_{1-x} \mathrm{Co}_{x}$ alloys, Phys. Rev. B 93, 224425 (2016).

[45] A. A. Mostofi, J. R. Yates, Y.-S. Lee, I. Souza, D. Vanderbilt, and N. Marzari, Wannier90: A Tool for Obtaining Maximally-Localised Wannier Functions, Comput. Phys. Commun. 178, 685 (2008).

[46] A. A. Soluyanov and D. Vanderbilt, Computing topological invariants without inversion symmetry, Phys. Rev. B 83, 235401 (2011).

[47] D. Gresch, G. Autès, O. V. Yazyev, M. Troyer, D. Vanderbilt, B. A. Bernevig, and A. A. Soluyanov, Z2pack: Numerical implementation of hybrid wannier centers for identifying topological materials, Phys. Rev. B 95, 075146 (2017).

[48] K. Parlinski, Z. Q. Li, and Y. Kawazoe, First-principles determination of the soft mode in cubic $\mathrm{ZrO}_{2}$, Phys. Rev. Lett. 78, 4063 (1997).

[49] A. Togo, F. Oba, and I. Tanaka, First-principles calculations of the ferroelastic transition between rutile-type and $\mathrm{CaCl}_{2}$-type $\mathrm{SiO}_{2}$ at high pressures, Phys. Rev. B 78, 134106 (2008).

[50] W. Ku, T. Berlijn, and C.-C. Lee, Unfolding FirstPrinciples Band Structures, Phys. Rev. Lett. 104, 216401 (2010).

[51] M. Tomić, H. O. Jeschke, and R. Valentí, Unfolding of electronic structure through induced representations of space groups: Application to Fe-based superconductors, Phys. Rev. B 90, 195121 (2014).

[52] J. Lander and J. Morrison, Surface reactions of silicon with aluminum and with indium, Surf. Sci. 2, 553 (1964).

[53] J. E. Northrup, $\operatorname{Si}(111) \sqrt{3} \times \sqrt{3}-A l:$ An Adatom-Induced Reconstruction, Phys. Rev. Lett. 53, 683 (1984).

[54] J. M. Nicholls, B. Reihl, and J. E. Northrup, Unoccupied surface states revealing the Si(111) $\sqrt{ } 3 \sqrt{ } 3-A l,-G a$, and -In adatom geometries, Phys. Rev. B 35, 4137 (1987).

[55] R. J. Hamers, Effects of coverage on the geometry and electronic structure of Al overlayers on Si(111), Phys. Rev. B 40, 1657 (1989).

[56] D. Xiao, W. Yao, and Q. Niu, Valley-Contrasting Physics in Graphene: Magnetic Moment and Topological Transport, Phys. Rev. Lett. 99, 236809 (2007).

[57] D. Xiao, G.-B. Liu, W. Feng, X. Xu, and W. Yao, Coupled Spin and Valley Physics in Monolayers of $\mathrm{MoS}_{2}$ and Other Group-VI Dichalcogenides, Phys. Rev. Lett. 108, 196802 (2012).

[58] C.-C. Liu, H. Jiang, and Y. Yao, Low-energy effective Hamiltonian involving spin-orbit coupling in silicene and two-dimensional germanium and tin, Phys. Rev. B 84, 195430 (2011).

[59] K. Song, D. Soriano, A. W. Cummings, R. Robles, P. Ordejn, and S. Roche, Spin proximity effects in graphene/topological insulator heterostructures, Nano Letters 18, 2033 (2018).

[60] P. Tang, P. Chen, W. Cao, H. Huang, S. Cahangirov, L. Xian, Y. Xu, S.-C. Zhang, W. Duan, and A. Rubio, Stable two-dimensional dumbbell stanene: A quantum spin hall insulator, Phys. Rev. B 90, 121408 (2014).

[61] D. Liu, A. G. Every, and D. Tománek, Continuum approach for long-wavelength acoustic phonons in quasitwo-dimensional structures, Phys. Rev. B 94, 165432 (2016).

[62] B. Peng, H. Zhang, H. Shao, Y. Xu, R. Zhang, and H. Zhu, The electronic, optical, and thermodynamic properties of borophene from first-principles calculations, J. Mater. Chem. C 4, 3592 (2016).

[63] W. Yu, C.-Y. Niu, Z. Zhu, X. Wang, and W.-B. Zhang, Atomically thin binary vv compound semiconductor: a first-principles study, J. Mater. Chem. C 4, 6581 (2016).

[64] P. Rivero, J.-A. Yan, V. M. García-Suárez, J. Ferrer, and S. Barraza-Lopez, Stability and properties of high-buckled two-dimensional tin and lead, Phys. Rev. B 90, 241408 (2014). 